\title{
Role of contextual imagery in associative recall
}

\author{
EUGENE WINOGRAD and DANIEL S. LYNN \\ Emory University, Atlanta, Georgia 30322
}

\begin{abstract}
The advantage of interactive over separation imagery in associative recall was markedly diminished when a unique imaginary context was provided for each imaged pair of words. Separation imagery was much more sensitive to contextual redundancy than was interactive imagery. Context was viewed as facilitating organization at encoding as well as affecting cue loading at retrieval. A three-element associative model is described.
\end{abstract}

For mental imagery to benefit associative learning, it is essential that the learner construct an image in which the referents of the two nouns to be associated are interacting. The strongest evidence for this statement comes from an ingenious experiment by Bower (1970) comparing interactive and separation imagery. Subjects who employed separation imagery showed no increase in associative recall compared to a verbal repetition control, while an interactive image group showed a much higher level of performance. Begg (1973, 1978) and Robbins, Bray, Irvin, and Wise (1974) found a similar outcome with concrete words, as did Neisser and Kerr (1973) when the words were embedded in sentences describing either interactive or separate pictorial frames. The research reported here shows that the mnemonic effects of separation imagery are intertwined with the imagined context in which the images of the word referents are set.

In Bower's (1970) report on the separation imagery manipulation, he says, "The instructions to Ss in this condition were to vividly imagine the $A$ and $B$ referents, but to keep them noninteracting, well separated in imaginal space, as though they were separate, still photographs on different walls of a room" (p. 530). An imaginary context is explicitly mentioned here, namely, a room containing walls on which images may be hung. Bower's description of instructions to subjects in the interactive imagery condition is that they "were told to visualize the objects denoted by the words of a pair and to imagine these interacting in some vivid way in an integrative scene" $(1970$, p. 531). Is there an imaginary context accompanying each interacting image, that is, a setting in which the interacting pair is placed? It is hard to say, but it is plausible to assume that, if subjects are placing their interactive images in backgrounds or contexts, these contexts are less alike, one to the other, than the identical background of the walled room for all the pairs in the separate imagery condition. If imagined contexts play a role in recall,

The authors are grateful to Martin M. Shapiro for his advice on data analysis. Reprints can be obtained from Eugene Winograd, Department of Psychology, Emory University, Atlanta, Georgia 30322. then it is possible that subjects using separation imagery were at a disadvantage in Bower's study in having a single context shared by all pairs. This assumption was tested here.

There are two published experiments clearly pointing to the participation of contextual factors when mental imagery is used to facilitate memory. In one experiment, Lesgold and Goldman (1973) asked subjects to form images of word triplets where one word of the triplet was the name of a location. This location word was shared by 10 triplets. On the basis of verbal descriptions by the subject of his constructed images, a score was obtained for the number of different specific contexts described for each location word. In tests of both recognition and recall, high positive correlations were found between memory and the number of different contexts described for each location word. Lesgold and Goldman (1973) argued that encoding uniqueness may account for much of the power of imagery mnemonics. In the Bower (1970) study described above, it is clear that placing mental images in the same imaginary context would lead to a high degree of contextual redundancy.

Petersen (1974), taking a different approach to the study of contextual factors, demonstrated that the rated imagery values of words had no effect on recall when associative strength between imaged words and the context for the compound image was controlled. Recall was accounted for only by the strength of the connection between words entering into the image and the context in which the image was placed. Indeed, Petersen suggests that previous work showing the greater retrieval potency of concrete over abstract words be reinterpreted as showing that concrete words are more likely to elicit the context in which the image is set.

Petersen's (1974) suggestion that memory for the noun pairs is mediated by memory for their context assigns a major role in recall to contextual factors. A similar outlook motivated the present research. Whereas Petersen was concerned with the role of contextual memory in accounting for differences in the memorability of concrete and abstract words, the focus of this research is on the role of memory for context in determining associative recall under interactive and 
separation imagery strategies. Perhaps the elevated recall associated with instructions to form interactive images is a consequence of the creation of a specific imaginal context that serves as an efficient mediator at the time of recall.

The approach taken in the studies presented here is to examine recall following separation and interactive memory instructions when the uniqueness of the contexts in which the word pairs are imaged is manipulated.

\section{EXPERIMENT 1}

The first experiment was exploratory in nature and sought to find if recall following separation imagery instructions would be affected by whether contexts were shared by all pairs or were unique for each pair. In addition, an interactive imagery group was tested without specification of any context.

\section{Method}

Subjects. There were 52 subjects of both sexes who were informally solicited by the junior author from Emory University dormitories. Twenty-two subjects served in the separation imagery/shared context (separate-shared) condition, 12 subjects served in the separation imagery/unique context (separateunique) condition, and 18 served in the interactive imagery condition.

Materials. Sixty concrete words were taken from the norms of Paivio, Yuille, and Madigan (1968) and combined into 30 pairs. A set of 30 locations was made up to serve as contexts. Some examples of contexts, with the context for interacting pairs given first in parentheses, are: a movie theater (with one or two screens), a soccer field (looking at one or both goals), sitting in a car (looking out of the front window or front and rear windows), at a baseball game (looking at home plate or home plate and second base), at a restaurant eating dinner (the table to the right or the table to the right and the table to the left). For interacting pairs, subjects were provided with one location within the setting (one screen or one goal, for example) while, for separate pairs, two locations within the same setting were provided (e.g., two movie screens or both soccer goals).

Procedure. A between-subjects design was used. All subjects were first told that the experiment was about visualization and their ability to form mental images. They were shown a 7-point scale for rating the vividness of their images and were instructed in its use. Nothing was said about a test of memory before the pairs of words were presented. Subjects were then read the instructions appropriate for their condition. The separate-shared subjects were told to imagine themselves standing in the middle of a room with four walls, two of which (the left and right walls) contained a window. For each pair of nouns, they were asked to image the referent of one noun in one of the windows and the referent of the second noun in the other window. The separateunique subjects were told that they would be given a new context for each pair of words. For example, for the room context they were told: "Picture yourself in a room surrounded by four walls. As you stand in the middle of the room, you look to your left and you can see one window, and as you look to your right, you can see another window. Place one of the objects in one window and another of the objects in the second window." The words were shown following the context description. The interactive imagery subjects were told to form an interacting image for each pair of words; nothing was said about context. All subjects rated each image on a 7-point rating scale where 1 represented high vividness. The noun pairs were shown together on cards for all groups.
Thirty pairs were presented to each subject with the first five pairs serving as practice. After the fifth pair, the experimenter stopped and engaged the subjects in a discussion of their images and ratings. The vividness scale was emphasized again, and any questions were answered. The 25 test pairs were then presented along with their contexts, where appropriate. Subjects were tested in groups of eight or less. The rate of presentation of the pairs was determined by how long it took the slowest subject to rate the vividness of his image for that pair. In Experiments 1 and 2, the interpair interval was inevitably longer for unique context than shared context groups because a new context had to be described for each pair. This confounding with spacing is equal for both interactive and separation imagery. Subjects were not explicitly instructed to keep successive images separate from each other in the manner described by Begg (1978). An unanticipated cued recall test followed immediately after presentation of the last pair. The first member of each word pair was shown on an index card, and subjects were instructed to write down that word as well as the word that had accompanied it at study. The test cards were shuffled for each experimental session. Scoring was strict in that synonyms were not scored as correct, but misspellings and pluralizations were counted as correct.

\section{Results and Discussion}

The mean proportion of words recalled was .10 for the separate-shared group, .45 for the separate-unique group, and .61 for the interactive imagery group. The respective standard deviations were $.13, .22$, and .20 . All differences between means were significant by $t$ test (the .05 level of confidence is used throughout this paper). The mean vividness ratings were 2.68 for the separate-shared group, 2.77 for the separate-unique group, and 3.43 for the interactive imagery group. The vividness ratings were not reliably different from each other $[F(2,49)=3.07]$. The important finding that led to the next two experiments is the powerful effect of the context manipulation on the separation imagery groups: Recall was 4.5 times greater when separate images were constructed in unique contexts than when they were all placed in the same setting. This led to a fuller investigation of the role of imaginary contexts in recall following separation and interactive imagery.

\section{EXPERIMENT 2}

In this experiment, a factorial design was used so that the effects of context could be examined for interactive as well as separation imagery. In addition, the usual interactive imagery instructions that do not mention context were read to a fifth group. In effect, Experiment 2 is a replication of Experiment 1 with the addition of two interactive imagery groups and tighter controls.

\section{Method}

Subjects. The subjects were 60 Emory undergraduates enrolled in introductory psychology classes who participated to fulfill a course requirement. There were 12 subjects assigned to each of five conditions in order of their appearance.

Procedure and Materials. Besides the addition of two new conditions, interactive imagery/shared context (interactive- 
shared) and interactive imagery/unique context (interactiveunique), the procedure of the first experiment was modified as follows. Subjects were tested individually; the list was shortened to 23 pairs, the first 3 of which were practice pairs; for half the subjects in each group, the order of presentation of the word pairs was reversed, but the contexts were presented to all subjects in the same order; and subjects were allowed up to $15 \mathrm{sec}$ during the recall test. Furthermore, each subject in the shared context conditions was given a different context in which to place his images; that is, in this condition, a given subject used the same context for the entire list, but each subject had a different context for his or her list. Since there were 12 subjects per group but 20 contexts, some contexts were not used for the shared context groups.

The instructions to the interactive-unique group were the same as those for the separate-unique group except for modifications required to make them sensible. For example, for the room context described in the Procedure section of Experiment 1, subjects in the interactive-unique group were told: "I want you to imagine yourself standing in a room surrounded by four walls, whereby as you stand in the middle of the room you can look to your left and see a window. Can you imagine this? If given the word pair 'duck' and 'match,' first call to mind the image of the context, in this case the room with the window; next, you would visualize the objects 'duck' and 'match' either in or out of the window interacting in some way. You may image, for instance, the duck sitting on the window sill with a match in its beak, striking the match against the wood, or maybe you might visualize the duck flying by the window with a lit match in its beak." Subjects in the interactive-shared group were told to use the same imaginary context for all of their pairs and to make the pairs interact in some way. The interactivecontrol group was instructed as in the first experiment with no mention made of any context. This group was included to serve as a reference point, since it is the most frequently used associative imagery condition. As in Experiment 1, the pair of words was presented together on an index card. The subjects rated the vividness of their images, and the experimenter did not present the next item until the vividness rating was circled. Also, the subjects were not told about a memory test until after presentation of the list. During the cued recall test, the experimenter shuffled the index cards bearing the cue word for each subject.

\section{Results}

The mean recall proportions and standard deviations for Experiment 2 are shown in Table 1. Two of the three values are essentially identical across Experiments 1 and 2 , in spite of some procedural differences, while recall for the separate-unique condition was higher in Experiment 2. Table 1 shows a marked interaction between imagery strategy and context. The results of a two-way analysis of variance on the recall frequencies (the data for the standard interactive imagery group are not included in this analysis) confirmed its significance $[F(1,44)=11.88]$. Both main effects were significant

Table 1

Mean Recall Proportions: Experiment 2

\begin{tabular}{|c|c|c|c|c|c|c|}
\hline \multirow[b]{3}{*}{ Imagery } & \multicolumn{6}{|c|}{ Context } \\
\hline & \multicolumn{2}{|c|}{ Shared } & \multicolumn{2}{|c|}{ Unique } & \multicolumn{2}{|c|}{ None } \\
\hline & Mean & SD & Mean & SD & Mean & SD \\
\hline $\begin{array}{l}\text { Separation } \\
\text { Interactive }\end{array}$ & $\begin{array}{l}.10 \\
.51\end{array}$ & $\begin{array}{l}.09 \\
.25\end{array}$ & $\begin{array}{l}.60 \\
.64\end{array}$ & $\begin{array}{l}.20 \\
.15\end{array}$ & .60 & .23 \\
\hline
\end{tabular}

as well [for imagery strategy, $F(1,44)=18.65$; for context, $F(1,44)=34.85, \quad M S e=13.271$. The data indicate that, while imaging pairs of nouns in the same context harms recall, the effect is largely confined to separation imagery. Indeed, cued recall was only about two items following the use of separation imagery for 20 pairs in the same imagined spatial setting. This is lower recall than would be expected with verbal repetition of the pairs (Bower, 1970). Yet, when separation imagery is utilized with a unique context for each pair, recall is at about the same level found when interacting imagery is used (no significant difference was found). This is powerful evidence for the sensitivity of separation imagery mnemonics to contextual factors. When interactive imagery is employed, the contribution of context is not great; it is noteworthy that recall is high with shared contexts as long as interacting imagery is used.

In related work, Begg and Anderson (1976) had sixth-grade children construct interactive images on the front blackboard or separate images on the side walls. In these shared context conditions, cued recall was $73 \%$ for interactive imagery and $21 \%$ for separation imagery, an outcome consistent with the data of Table 1 for shared contexts. With adults, Begg (1978, Experiment 1) found only $8 \%$ recall for separation imagery, a result in close agreement with the $10 \%$ value found in Experiments 1 and 2 here. It is clear that temporal contiguity alone is not sufficient to produce even a modest level of associative recall when separate images are constructed in a redundant context.

The mean vividness ratings for the four groups in the cells making up the factorial design varied between 2.77 and 3.01 and showed no statistically significant effects.

\section{Discussion}

A useful and relatively simple way to conceptualize what is happening is presented here. We start with the assumption that there are three cognitive elements stored, the representations of the two words, A and B, plus a representation of context, $C$. What differentiates separation from interactive imagery is that $A$ and $B$ are not directly associated when separation imagery is involved, but are directly associated when an interactive image has been formed. The three elements and their hypothesized interconnections are shown in Figure 1 for both separation and interactive imagery. According to this representation, the only route from $A$ to $B$ for separation imagery lies through the recall of context, which may operate as a control element (Estes, 1972). We suggest that recall is very poor for the separateshared condition because the context element is overloaded when the same context is used for 40 items. For the separate-unique condition, if the subject gets from $\mathrm{A}$ to $\mathrm{C}$, he is in a favorable position on the cueloading function, since each context is connected to one and only one B term (see Watkins \& Watkins, 1975, 


\section{SEPARATION IMAGERY}

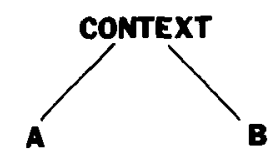

INTERACTIVE IMAGERY

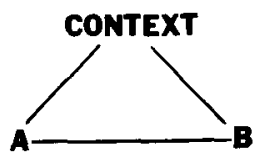

Figure 1. Theoretical relationships among the three memory elements corresponding to context, Word A, and Word B when studied with separation imagery and with interactive imagery.

for a fuller exposition of the role of cue loading in retrieval). With interactive imagery, even when the context element is overloaded, the subject can still retrieve $B$ directly from $A$ since $A$ is a unique cue for B. Experiment 3 was designed to explore this line of reasoning further.

\section{EXPERIMENT 3}

In Experiment 3, subjects were cued with either the A term or with the context, C, and asked to recall both of the other items. The examination of recall with the context cue provided and the request for retrieval of both of the other items are new to this experiment. When recall of two elements is demanded, richer information is available to the experimenter than when only a single element is required. Thus, one prediction that follows from the simple model depicted in Figure 1 is that, when cued with Word A following separation imagery, the probability of recalling $B$ given failure to recall context [i.e., $\mathrm{P}(\mathrm{B} \mid \overline{\mathrm{C}})]$ should approach zero.

The goal of Experiment 3 was to examine response patterns that might support or disconfirm this model. To this end, separation and interactive imagery were studied only with unique contexts. While these two conditions did not differ in recall in Experiment 2, the design of Experiment 3 allows for potentially interesting different patterns of responses to appear.

\section{Method}

Design and Procedure. Two encoding conditions were studied in this experiment, separation imagery with unique contexts and interacting imagery with unique contexts. There were two kinds of retrieval cues provided at test, the $A$ term or context (C). Thus, there were four distinct groups: separationunique/Cue $\mathrm{A}$, separation-unique/Cue $\mathrm{C}$, interactive-unique/ Cue $A$, and interactive-unique/Cue $C$. The experiment was conducted in two sessions with subjects enrolled in introductory psychology classes at the Georgia Institute of Technology. One group $(n=28)$ was read separation imagery instructions and the other group $(n=28)$ was read interacting imagery instructions. The instructions were identical to those used in Experiment 2, with the exception that the subjects were told that their memory would be tested later, although the manner of testing was not specified. Intentional learning instructions were introduced here because the study had been advertised as about memory. With the exception of Neisser and Kerr (1973), all of the work comparing separation and interactive imagery cited earlier used intentional learning instructions. The same materials were used, but since large groups were tested at once, all subjects received the contexts and their associated word pairs in the same order (this order had been used for half the subjects in Experiment 2). As in Experiment 2, vividness ratings were requested. The experimenter read the contexts followed by the pair of words. Approximately $10 \mathrm{sec}$ were allowed before the next pair was read, during which time the subjects made their vividness ratings. There were 3 practice pairs and then the 20 pairs that were scored.

Following the presentation of the list of contexts and word pairs, the experimenter distributed the recall test booklet, consisting of two sheets. On the front page were instructions. The next page listed either the $20 \mathrm{~A}$ words or 20 contexts as cues, followed by two spaces for recall. The cue order was the same as the study order. Contexts were described in a few words [e.g., "at the soccer field with the (two) goal(s)," "sitting in the car looking out the window(s)," "at a baseball game," "in a restaurant eating dinner")]. Word-cue and context-cue booklets were alternated. For separation imagery, there were 15 subjects cued with contexts and 13 cued with words; for interacting imagery, there were 13 subjects cued with contexts and 15 cued with words. Ample time was allowed for subjects to complete recall. In scoring word recall, the same criteria used for Experiments 1 and 2 were followed; for context recall, a more lenient criterion was adapted. For example, "sitting at a table eating" or "in the restaurant" or "eating dinner" were scored as correct recall of the context described by the experimenter as "in a restaurant eating dinner."

\section{Results and Discussion}

Word cues. The recall proportions for Experiment 3, with standard deviations, are shown in Table 2 . It can be seen that, when cued with Word A, the probability of recall of Word $B$ and of context (C) was higher with interactive imagery. However, neither difference was statistically significant [ for recall of $B, t(26)=1.92$; for recall of $C, t(26)=.69]$. The model shown in Figure 1 leads one to expect higher recall for $\mathrm{B}$, given Cue A, with an interactive image, since there are two routes from $A$ to B compared with only one route (through C) for separation imagery. This prediction was not confirmed in either experiment, although in both cases recall was higher for the group using interaction.

It should be noted that recall of context was slightly,

Table 2

Recall Proportions: Experiment 3

\begin{tabular}{|c|c|c|c|c|c|c|c|c|}
\hline \multirow[b]{3}{*}{ Imagery } & \multicolumn{4}{|c|}{ Word Cue } & \multicolumn{4}{|c|}{ Context Cue } \\
\hline & \multicolumn{2}{|c|}{$p(B)$} & \multicolumn{2}{|c|}{$\mathrm{p}(\mathrm{C})$} & \multicolumn{2}{|c|}{$\mathrm{p}(\mathrm{B})$} & \multicolumn{2}{|c|}{$\mathrm{p}(\mathrm{A})$} \\
\hline & Mean & SD & Mean & SD & Mean & SD & Mean & SD \\
\hline $\begin{array}{l}\text { Separation } \\
\text { Interactive }\end{array}$ & $\begin{array}{l}.55 \\
.70\end{array}$ & $\begin{array}{l}.21 \\
.20\end{array}$ & $\begin{array}{l}.61 \\
.66\end{array}$ & $\begin{array}{l}.24 \\
.19\end{array}$ & $\begin{array}{l}.74 \\
.74\end{array}$ & $\begin{array}{l}.19 \\
.16\end{array}$ & $\begin{array}{l}.67 \\
.80\end{array}$ & $\begin{array}{l}.26 \\
.10\end{array}$ \\
\hline
\end{tabular}


but not significantly, higher with interactive imagery: There was no evidence of a stronger word-to-context association when separate images were made. This is not incompatible with the model since, with in teractive imagery, context may be retrieved sometimes via a path leading from $\mathrm{A}$ to $\mathrm{B}$ to $\mathrm{C}$, a path not available with separation imagery.

Another way to examine the data is to look at the joint probability of each pair of outcomes. That is, subjects could recall zero, one, or two elements on each recall attempt. These probabilities are presented in Table 3 separately for each type of cue. Consider only the left-hand part of Table 3. It can be seen that, with the word cues, recall is fragmented (only one element is recalled) for less than $20 \%$ of the pairs. Conditional probabilities were computed from these values. As noted above, the value of $\mathrm{P}(\mathrm{B} \mid \overline{\mathrm{C}})$ should approach zero when separation imagery is used, according to the state of affairs represented in Figure 1. The obtained value of $\mathrm{P}(\mathrm{B} \mid \overline{\mathrm{C}})$ was .07 for the separation imagery condition, compared to .32 for interactive imagery. In other words, when the referents of words are not represented as interacting in some way, it is highly unlikely that the second word will be recalled when information about the context is not accessible. On the other hand, when the $A$ and $B$ terms are represented as interacting, subjects can get from $A$ to $B$ on one-third of the occasions when contextual information is not retrieved, an outcome accommodated by the pathway between $A$ and $B$ in the right-hand part of Figure 1.

Context cues. The recall proportions, $\mathrm{P}(\mathrm{A})$ and $P(B)$, to the context cue are presented in the right-hand part of Table 2. Recall of the B term was equal for both imagery conditions, and the superiority observed for recall of $\mathrm{A}$ with interactive imagery was not significant $[t(26)=1.76]$. The right-hand part of Table 3 gives the joint recall probabilities when context cues were provided. It may be noted that, for separation imagery, the probability of fragmented recall was .25 , compared with .12 for interactive imagery.

Tests of independence. With the data of Experiment 3 , one can ask whether recall of the two elements for a given cue is independent. The values in the second and fifth columns of Table 4 are the predicted values for the joint recall of both elements on the hypothesis that recall of one is statistically independent of recall of the other. In each case, the observed joint probability exceeds the expected probability. To test the significance of the departure from independence, instead of pooling results from individuals and testing the pooled result, the results of each individual were tested

Table 3

Joint Probabilities of Recall Outcomes: Experiment 3

\begin{tabular}{lccccccccc}
\hline & \multicolumn{3}{c}{ Word Cue } & & \multicolumn{4}{c}{ Context Cue } \\
\cline { 2 - 5 } \cline { 6 - 9 } Imagery & $\mathrm{B}, \mathrm{C}$ & $\mathrm{B}, \overline{\mathrm{C}}$ & $\overline{\mathrm{B}}, \mathrm{C}$ & $\overline{\mathrm{B}}, \overline{\mathrm{C}}$ & $\mathrm{A}, \overline{\mathrm{B}}$ & $\mathrm{A}, \overline{\mathrm{B}}$ & $\overline{\mathrm{A}}, \mathrm{B}$ & $\overline{\mathrm{A}}, \overline{\mathrm{B}}$ \\
\hline Separation & .53 & .03 & .08 & .36 & .58 & .09 & .16 & .17 \\
Interactive & .60 & .11 & .07 & .23 & .71 & .09 & .03 & .16 \\
\hline
\end{tabular}

Table 4

Comparison of Observed (O) Joint Probabilities and Those Predicted (P) if Recall is Independent

\begin{tabular}{cccccccr}
\hline & \multicolumn{3}{c}{ Word Cue } & & \multicolumn{3}{c}{ Context Cue } \\
\cline { 2 - 4 } Imagery & $\mathrm{P}(\mathrm{B}+\mathrm{C})$ & $\mathrm{P}$ & $\mathrm{z}$ & & $\mathrm{O}$ & $\mathrm{P}(\mathrm{A}+\mathrm{B})$ & $\mathrm{z}$ \\
\hline Separation & .53 & .33 & -9.07 & .58 & .50 & 1.64 \\
Interactive & .60 & .46 & -2.10 & .71 & .59 & -6.07 \\
\hline
\end{tabular}

separately, and the statistical results were pooled. Maxwell (1961) describes a procedure for combining probabilities obtained from separate experiments into a single test of statistical significance. The method used here is directly analogous to the method presented by Maxwell (1961, Chapter 5). For this application, each subject is considered as a separate experiment. A 2 by 2 matrix was constructed for each subject in which the rows were the frequencies of recall of $A$ and failure to recall $A$ and the columns were the frequencies of recall of $B$ and failure to recall $B$ (an identical procedure was followed for recall of $B$ and C). An exact probability was obtained for each subject's table with Fisher's exact test, and this probability was converted to a $\mathrm{z}$ score. For each group, a $\mathrm{z}$ score was then obtained by taking the sum of the individual $z$ scores and dividing by the square root of the number of subjects. This group score is, in turn, a $\mathrm{z}$ score and, if significant, may be taken as evidence for significant departure from independence in recall of the two elements in question.

The values of $\mathrm{z}$ for each group are shown in Table 4 . The only condition for which the hypothesis of statistical independence cannot be rejected is when the context cue was presented following separation imagery. This outcome is compatible with the model, since A and $\mathrm{B}$ are not directly connected when separate images were constructed.

Vividness. The mean vividness ratings were 3.25 and 3.61 with separation and interactive imagery instructions, respectively. The difference was not significant.

\section{GENERAL DISCUSSION}

The central finding of this research is that imagined contexts can play a significant role in determining the relative mnemonic effectiveness of different memory strategies. Specifically, the results of the first two studies showed that, following separation imagery, recall was four to six times greater when each pair of words was placed in a unique, rather than a shared, context. The power of imagined contexts is attested to by the finding that contextual uniqueness markedly attenuated the well documented superiority of interactive over separation imagery. At the same time, it is clear that the absolute effectiveness of interactive imagery was affected little by manipulation of context. A pair-specific context does not seem to be essential for recall when interactive images are formed. Therefore, the hypothesis that interactive imagery derives its 
potency from the construction of context-specific images received little support.

The relationship between context and imagery strategy found here may be interpreted in terms of factors operating at both encoding and retrieval. With respect to encoding, Bransford and McCarrell (1974) argue that mental imagery is simply one way of constructing a "meaningful relation" between concrete words, and they discuss imagery in terms dictated by their emphasis on the importance of comprehension in cognition. The finding that supplying a unique context facilitates the recall of separate images could be interpreted as a demonstration that context aids in finding a meaningful relation between otherwise unrelated words. To handle the findings of the present research, one has only to further assume that such comprehension is more likely to occur with a unique rather than a shared context. Bower $(1970,1972)$ interprets the superior performance he obtained with interactive imagery along much the same lines, saying, "I am convinced that associating meaningful terms is largely a matter of relating them in some organization" (1972, p. 86), a thesis that, he points out, has been advocated by Gestalt thinkers such as Asch (1969). Begg $(1973,1978)$ has offered a similar interpretation.

Another way of looking at the present findings emphasizes events taking place at retrieval. As pointed out earlier, there is greater cue loading when contexts are shared than when they are unique to a word pair. We agree with Watkins and Watkins (1975) that "the efficiency of a functional retrieval cue in effecting recall of an item declines as the number of items it subsumes increases" (p.443). As shown in Figure 1, we assume that, with separation imagery, recall of $B$ to Cue $A$ requires implicit retrieval of contextual information that, in turn, serves as the functional retrieval cue for $B$. When the context is shared by 20 word pairs, its cuing efficiency is drastically reduced compared with the case when it is connected to only 1 pair. With interactive imagery, on the other hand, the direct pathway between $\mathrm{A}$ and $\mathrm{B}$ allows $\mathrm{A}$ to be the functional cue for $B$ in most cases and, to the point, $\mathrm{A}$ has a loading of unity since no $\mathrm{A}$ term was repeated.

We do not see these two interpretations, one emphasizing encoding and the other retrieval, as conflicting; rather, we see both as applicable to the present case. The provision of an imaginary context is regarded as a sufficient condition for establishing A-B organization; however, retrieval problems arising from overloading of the retrieval cue (or control element) prevent successful retrieval of information about $B$ when separate images are constructed in a redundant context. With favorable encoding and retrieval conditions, as is the case when each pair has its own context, separation imagery results in adequate recall. A similar argument emphasizing the advantages of encoding uniqueness in retrieval has been made by Lesgold and Goldman (1973).
We conclude with a methodological point. In research concerned with the role of contextual factors in memory, manipulation of context has often involved the cumbersome procedure of transporting subjects from one environment to another. Environmental contexts have ranged from two experimental rooms (e.g., Strand, 1970) to underwater settings (Godden \& Baddeley, 1975). The present work raises the possibility that manipulation of context with imagery instructions may be undertaken fruitfully with some gain in experimental flexibility.

\section{REFERENCES}

Asch, S. Reformulation of the problem of association. American Psychologist, 1969, 24, 92-102.

BEGG, I. Imagery and integration in the recall of words. Canadian Journal of Psychology, 1973, 27, 159-167.

BEGG, I. Imagery and organization in memory: Instructional effects. Memory \& Cognition. 1978, 6, 174-183.

BEGG, I., \& ANDERson, M. C. Imagery and associative memory in children. Journal of Experimental Child Psychology, 1976, 21, 480-489.

BowER, G. H. Imagery as a relational organizer in associative learning. Journal of Verbal Learning and Verbal Behavior, 1970, 9, 529-533.

BowER, G. H. Mental imagery and associative learning. In L. W. Gregg (Ed.), Cognition in learning and memory. New York: Wiley, 1972.

Bransford, J. D., \& McCarrell, N. S. A sketch of a cognitive approach to comprehension: Some thoughts about understanding and what it means to comprehend. In W. B. Weimer $\&$ D. S. Palermo (Eds.), Cognition and the symbolic processes. Hillsdale, N.J: Erlbaum, 1974.

Estes, W. K. An associative basis for coding and organization in memory. In A. W. Melton \& E. Martin (Eds.), Coding processes in human memory. New York: Wiley, 1972.

Godden, D. R., \& Baddeley, A. D. Context-dependent memory in two natural environments: On land and underwater. British Journal of Psychology, 1975, 66, 325-332.

LesGold, A. R., \& Goldman, S. R. Encoding uniqueness and the imagery mnemonic in associative learning. Journal of Verbal Learning and Verbal Behavior, 1973, 12, 193-202.

Maxwell, A. E. Analysing qualitative data. New York: Wiley, 1961.

NeIsser, U., \& KERR, N. Spatial and mnemonic properties of visual images. Cognitive Psychology, 1973, 5, 138-150.

Paivio, A., Yuille, J. C., \& Madigan, S. A. Concreteness, imagery, and meaningfulness values for 925 nouns. Journal of Experimental Psychology Monograph Supplement, 1968, 76(Whole No. 1), 1-25.

Petersen, R. C. Imagery and cued recall: Concreteness or context? Journal of Experimental Psychology, 1974, 102, $841-844$

Robbins, D., Bray, J. F., Irvin, J. R., \& Wise, P. S. Memorial strategy and imagery: An interaction between instructions and rated imagery. Joumal of Experimental Psychology, 1974, 102, 706-709.

StRAND, B. Z. Change of context and retroactive inhibition. Journal of Verbal Learning and Verbal Behavior, 1970, 9, 202-206.

Watkins, O. C., \& Watkins, M. J. Buildup of proactive inhibition as a cue-overload effect. Journal of Experimental Psychology: Human Learning and Memory, 1975, 104, 442-452.

(Received for publication August 3, 1978; revision accepted December $12,1978$. 